\title{
Safety Experience During Real-World Use of Injectable Artesunate in Public Health Facilities in Ghana and Uganda: Outcomes of a Modified Cohort Event Monitoring Study (CEMISA)
}

\author{
H. Hilda Ampadu ${ }^{1,2}$ - Alexander N. O. Dodoo ${ }^{1,7}$ - Samuel Bosomprah ${ }^{3}$. \\ Samantha Akakpo $^{4} \cdot$ Pierre Hugo $^{4} \cdot$ Helga Gardarsdottir $^{2}$ - H. G. M. Leufkens ${ }^{2}$ • \\ Dan Kajungu ${ }^{5} \cdot$ Kwaku Poku Asante ${ }^{6}$
}

Published online: 25 April 2018

(C) The Author(s) 2018

\begin{abstract}
Introduction Injectable artesunate (Inj AS) is the World Health Organization (WHO)-recommended product for treating severe malaria. However, despite widespread usage, there are few published safety studies involving large populations in real-world settings. In this study, we sought to assess the incidence of common adverse events (AEs) following the intake of Inj AS in real-life settings.

Methods This is a modified cohort event monitoring study involving patients who were administered with Inj AS at eight sites (four each in Ghana and Uganda) between May and December 2016. Patients were eligible for inclusion if they had severe/complicated malaria and were able and willing to participate in the study. Eligible patients were followed up by telephone or hospital or home visit on Days
\end{abstract}

Alexander N. O. Dodoo

adodoo@gsa.gov.gh

1 African Collaborating Centre for Pharmacovigilance, P. O. Box LT282, Accra, Ghana

2 Utrecht Institute for Pharmaceutical Sciences, Utrecht University, Utrecht, The Netherlands

3 Department of Biostatistics, School of Public Health, University of Ghana, Accra, Ghana

4 Medicines for Malaria Venture, Geneva, Switzerland

5 Makerere University Centre for Health and Population Research, Kampala, Uganda

6 Kintampo Health Research Centre, Kintampo, Ghana

7 University of Ghana School of Medicine and Dentistry, Accra, Ghana
7, 14, 21 and 28 after drug administration to document AEs and serious AEs (SAEs). Patients were also encouraged to report all AEs at any time during the study period. The Kaplan-Meier method was used to estimate the proportion of patients with any AEs by end of Day 28. Causality assessment was made on all AEs/SAEs using the WHO/ UMC (Uppsala Monitoring Centre) causality method.

Results A total of 1103 eligible patients were administered Inj AS, of which 360 patients were in Ghana and 743 in Uganda. The incidence of any AE by the end of follow-up among patients treated with AS was estimated to be $17.9 \%$ (197/1103) (95\% confidence interval [CI] 15.8-20.3). The median time-to-onset of any AEs was 9 days (interquartile range $(\mathrm{IQR})=4,14)$. The top five AEs recorded among patients treated with AS were pyrexia (3.5\%), abdominal pain $(2.5 \%)$, diarrhoea $(1.7 \%)$, cough $(1.5 \%)$ and asthenia (1.5\%). Most of these top five AEs occurred in the first 14 days following treatment. Regarding the relatedness of these AEs to Inj AS, $78.9 \%$ of pyrexia (30/38), $63.0 \%$ of pain (17/27), $68.4 \%$ of diarrhoea (13/19), $85.5 \%$ of cough $(14 / 16)$ and $75.0 \%$ of asthenia (12/16) were assessed as 'possibly' related. There were 17 SAEs including 13 deaths. Two of the deaths are 'possibly' related to Inj AS, as were three non-fatal SAEs: severe abdominal pain, failure of therapy and severe anaemia.

Conclusion The incidence of common AEs among patients treated with Inj AS in real-world settings was found to be relatively low. Future studies should consider larger cohorts to document rare AEs as well.

ClinicalTrials.gov Identifier NCT02817919. 


\section{Key Points}

Injectable artesunate (Inj AS) is a life-saving medicine used to treat severe malaria.

There are few data on the safety of Inj AS when used in real-world settings, though it has been shown to be well-tolerated in clinical trials.

Safety data obtained from public health facilities in Ghana and Uganda support the safety findings from clinical trials and provide additional evidence for continued use of Inj AS in severe malaria.

\section{Introduction}

Severe malaria is a life-threatening condition responsible for a significant part of the 445,000 global malaria deaths that occurred in 2016 alone [1]. When not treated, the case fatality rate for severe malaria can be very high. Severe malaria is the harshest form of the disease. In addition to the symptoms of uncomplicated malaria such as fever, parasitaemia and malaise, severe malaria also manifests with one or more of the following: severe anaemia, acute renal failure, respiratory oedema, hypoglycaemia or coma. Published fatality rates for severe malaria vary widely due to study design, treatment practices and patient types. Fatality rates are typically around $16-20 \%$ but rates as low as $2 \%$ and high as $100 \%$ have been reported [2]. With prompt and effective treatment, case fatality rates can fall as low as $10 \%$ [2] or below. The current edition of the World Health Organization (WHO) Guidelines for the Treatment of Malaria (2015, third edition) [3] recommends injectable artesunate (Inj AS) (ATC [Anatomical Therapeutic Chemicall code P01BE03) as the treatment of choice for severe malaria. The SEAQUAMAT (South East Asian Quinine Artesunate Malaria Trial) [4] and AQUAMAT (Artesunate Versus Quinine in the Treatment of Severe Falciparum Malaria in African Children) [5] studies showed reductions in fatality of $34.7 \%$ and $22.5 \%$, respectively, when Inj AS was used to treat severe malaria instead of injectable quinine. In these studies, the use of Inj AS was also associated with fewer adverse events (AEs) than quinine. Systematic reviews $[6,7]$ have also demonstrated lower case fatality rates and lower AE profiles with Inj AS than with quinine. For years, parenteral quinine remained the main drug for treating severe malaria, but its usage is associated with problems in reconstitution and administration [8]. Quinine needs to be administered slowly as a constant intravenous (IV) infusion, a process which is difficult in most settings. It may also be given intramuscularly (IM) but IM administration is associated with erratic availability and poor clinical outcomes. In addition to these, the use of quinine is associated with several AEs including cinchonism, rashes, rare cardiotoxicity, deafness, hypoglycaemia, dizziness, blindness and even death $[9,10]$. These factors prompted the WHO policy change and subsequent recommendation for the use of Inj AS for treating severe malaria.

The current data on the efficacy and clinical safety of Inj AS have all been obtained in well-controlled clinical trials or during operational research [6,11-15]. The recruitment of patients in such settings is controlled and patient followup and management is stringent in these studies; hence, safety information obtained may not reflect what occurs in real life. There is a dearth of information on the safety of Inj AS when used in real-world (post-approval, routine healthcare practice) settings even though a signal-postartesunate delayed haemolysis (PADH) - has been raised following identification of a number of delayed haemolysis cases after treatment with Inj AS [16-18].

Inj AS is an extremely important life-saving product in the treatment of severe malaria across all 91 malaria-endemic countries and across all malaria transmission zones $[1,19]$. It is used extensively in imported or traveller's malaria in non-endemic countries, where it has been associated with very high reduction in mortality with few reports of drug-related AEs [20]. Despite the assurance given by the available studies on the safety of Inj AS, the absence of strong pharmacovigilance systems in countries that use millions of doses of the product annually makes it necessary to undertake appropriate post-authorisation studies in order to better understand its actual safety profile when used in real-world settings. This study was therefore conceived to obtain safety data in relation to Inj AS when used in real-world settings in public health facilities in two African countries where severe malaria may or may not be properly diagnosed (microscopy; rapid diagnostic tests [RDTs]; laboratory measurement of haemoglobin [Hb]) and where facilities for monitoring and follow-up are variable.

The specific objective of the study was to determine the incidence of any AEs that occur up to 28 days after administration of Inj AS for the treatment of severe/complicated malaria during the normal course of clinical practice in the participating health facilities. The findings from this study should contribute to the WHO global individual case safety report (ICSR) database VigiBase ${ }^{\mathrm{TM}}$ and facilitate quicker identification of safety signals. Currently, VigiBase ${ }^{\mathrm{TM}}$ has very little data from Africa that includes data on antimalarials [21, 22]. 


\section{Methods}

\subsection{Study Design, Sites and Patient Recruitment}

This was a prospective, longitudinal, modified cohort event monitoring study in sub-Saharan Africa (CEMISA) which utilises the principles of prescription event monitoring [23] but with cohorts smaller than the minimum 10,000 patients. The study recruits patients in secondary care settings similar to the approach adopted in specialised cohort event monitoring [24]. In this study, the cohort consisted of patients who were prescribed Inj AS for presumed or diagnosed severe malaria between May 2016 and December 2016 in two countries (Ghana and Uganda). The study was undertaken in four public health facilities in Ghana (Princess Marie Louise Hospital and Ridge Hospital, Accra; Kintampo Municipal Hospital, Kintampo and Agogo Medical Research Hospital, Agogo) and four public health facilities in Uganda (Mubende Regional Referral Hospital, Mubende; Jinja Referral Hospital, Jinja; Lira Regional Referral Hospital, Lira and Kagadi Hospital, Kagadi).

Patients were eligible for inclusion if they had severe/complicated malaria (Plasmodia of any species) presumed or diagnosed as per national policies and health facility practice/protocol [3]; if they were able and willing to participate in the study; and if they agreed to the schedule for follow-up contact or home visits. Patients were excluded if they had a serious concurrent illness. All eligible patients gave informed consent. For children, informed consent was obtained from parents or a caregiver/guardian.

Case Report Forms (CRFs) were used to record data on each study subject during the study as defined by the protocol. All events that happened in the study were fully documented in the CRF. The CRFs consisted of the day 1A form, drug administration form, follow-up forms (Days 7, 14, 21 and 28), AE form, SAE form and the end of study form. The day $1 \mathrm{~A}$ form served as the enrolment form on the first day of the study. It was the form used after the participant or representative signed the informed consent to record demographic, medical history and laboratory data. The drug administration form was used to record the drug under investigation administered to the participant and all other concomitant medications. The AE form was used to record all AEs and the SAE form was used to record events that met the seriousness criteria. The Day 7 to Day 28 follow-up forms were used to record the participant's current health status and any new concomitant medications. Finally, the end of study form was used to record the primary reason for the termination from the study. A completed CRF after going through the various validations and data quality checks is then prepared for entry into the study database.

Patients were followed up to document the occurrence of any AEs using standard questions on the follow-up CRFs. Patients were followed up by telephone or hospital or home visit, when possible, on Days 7, 14, 21 and 28 after drug intake (index date) and were asked to report all AEs at any time during the 28-day follow-up period. The 28-day follow-up period was adopted in line with the follow-up period adopted for malaria clinical trials [25] as well as previous studies on the safety of antimalarials [26]. No attempt was made to intervene in routine care of any of the recruited patients in the study apart from monitoring the safety of the antimalarial agents administered by the treating clinicians by collecting data from consenting patients directly and sometimes also from their clinical notes. Hb levels, when measured, were also recorded. Any patient with AEs was managed in line with existing standard of care in each of the participating facilities.

\subsection{Definitions}

The following definitions are based on the European Union's Guidelines on Good Pharmacovigilance Practice.

\subsubsection{Adverse Event (AE)}

An AE is any untoward medical occurrence in a clinical investigation participant temporally associated with the use of a medicinal product, whether or not considered related to the medicinal product. An AE can therefore be any unfavourable and unintended sign (including an abnormal laboratory finding), symptom or disease (new or exacerbated) temporally associated with the use of a medicinal product.

\subsubsection{Serious AE (SAE)}

An SAE means an AE that results in death, is life-threatening, requires in-patient hospitalisation or prolongation of existing hospitalisation, results in persistent or significant disability or incapacity, or is a congenital anomaly/birth defect.

\subsection{Data Collection, Drug Prescription and Dosing}

Routine clinical practice in malaria-endemic countries requires immediate treatment of patients with suspected clinical malaria in line with WHO Guidelines for the Treatment of Malaria [3]. Treatment of severe malaria occurs usually in in-patient settings. All study participants were recruited from hospitals. The implication for this study, therefore, was that most patients had started 
treatment before being recruited into the study. IM/IV artesunate was administered as per normal practice in the treating institutions. The actual dosage and duration of treatment as well as any concomitant medications were extracted from the patient's clinical notes and recorded on the study CRFs. The WHO recommendation [3] for treating severe malaria is to "treat all adults and children with severe malaria (including infants, pregnant women in all trimesters, lactating women) with intravenous or intramuscular artesunate for at least $24 \mathrm{~h}$. Once a patient has received at least $24 \mathrm{~h}$ of parenteral therapy and can tolerate oral therapy, complete treatment with 3 days of ACT [artemisinin-based combination therapy]". We also collected data on mode of malaria diagnosis (i.e. clinically, microscopically or by the use of RDTs). Other co-variables collected included laboratory investigations conducted (including $\mathrm{Hb}$ measurements). Data were entered, managed and stored in a specially created version of MedSpina ${ }^{\mathrm{TM}}$, an in-house electronic health records system that allows clinicians and other health workers to collect patients' data, including laboratory results, to facilitate patient care.

\subsection{Outcome and Causality Measurement}

The outcomes measured in the study were all AEs temporarily associated with the intake of Inj AS, including deaths and other SAEs. Assessment of causality included, where available, the level of parasitaemia as well as any concomitant medications administered. Since this was a non-interventional study, there was no systematic laboratory investigation to document AEs. However, all events reported or available in the patient's notes, including laboratory data, were extracted and recorded in the CRFs. Using the WHO/UMC (Uppsala Monitoring Centre) causality method, a physician and a pharmacist not involved in the direct care of the participants assessed the relatedness and causal link of the medicine to the AEs.

\subsection{Sample Size Calculation}

The study was powered to estimate the incidence of AEs with a certain level of precision in Ghana and Uganda. We assumed that the incidence of any AEs in the Ghanaian and Ugandan population was, on average, $20 \%$. We therefore required a total of 3164 patients in the two countries to produce a two-sided $95 \%$ confidence interval (CI) for the ratio of population proportions with a width that is equal to 0.200 when the estimated sample proportion decreases to 0.12 and the ratio of the sample proportions is 0.60 . Due to available funding, we planned to enrol a cumulative sample size of 1000 patients receiving Inj AS from all participating countries in the first part of this study with the additional number of 2164 expected in the second part. The 1000 patients produces a two-sided $95 \%$ CI with a width equal to 0.050 when the incidence of any $\mathrm{AE}$ is $20 \%$ as we have assumed.

\subsection{Data Analysis}

We summarized patients' characteristics using proportion (nominal scale variables) and mean or median (interval scale variables). We calculated the incidence of any AEs as the total number of any AEs recorded by end of follow-up divided by the total number of patients treated with AS and who completed the study. The Kaplan-Meier method was used to estimate the proportion of patients with any AEs by the end of Day 28. The date of treatment was considered as the origin (i.e. the date the patient was at risk of any $\mathrm{AE}$ ). The patient was censored at the date the patient was last seen (i.e. lost to follow-up) or at the end of the study without any AE.

Since the study was designed to have a cumulative sample size of 1000 patients on Inj AS from all participating countries (Part 1), with the eventual number of 3164 expected in Part 2, we considered site (country) as a fixed effect and therefore we did not present results for each country. All analyses were performed using STATA ${ }^{\circledR} 14$ MP (StataCorp, College Station, TX, USA). Case summaries were also presented for all SAEs, including deaths and their relatedness to Inj AS as well as the causality assessment gradings.

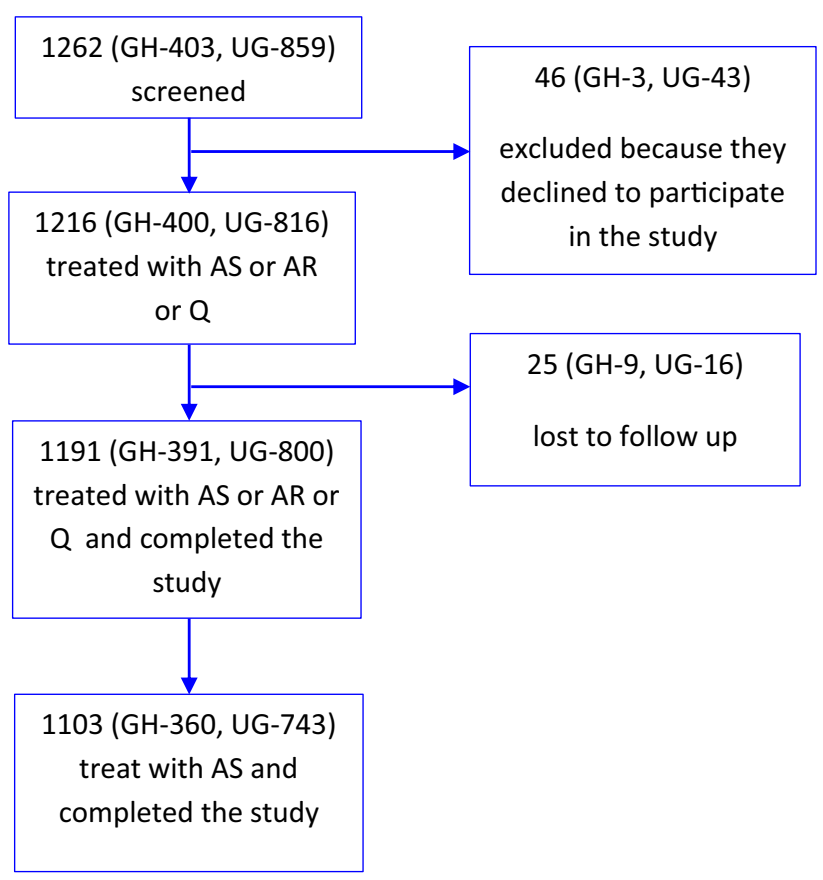

Fig. 1 Patient flow. $A E$ adverse event, $A R$ artemether, $A S$ artesunate, $G H$ Ghana, $Q$ quinine, $U G$ Uganda 


\section{Results}

\subsection{Characteristics of Participants and Treatment Received}

A total of 1262 patients were screened, of whom 46 were excluded for declining to participate in the study (Fig. 1). Of the 1216 eligible patients, 25 were lost to follow-up and 88 were treated with either artemether or quinine, making them ineligible for analysis. There were 1103 patients who were treated with Inj AS (360 in Ghana and 743 in Uganda) (Table 1) and completed the study. The median age of patients was 3.9 years (interquartile range $[\mathrm{IQR}]=2.1,9$ ) and the median weight was $13 \mathrm{~kg} \quad(\mathrm{IQR}=10,20)$ (Table 1).

\subsection{Patient Follow-Up and Recording of Haemoglobin Readings}

Most patients were followed-up by way of telephone calls. Of the 1103 individuals treated with Inj AS, 894 (81.1\%) were followed up by telephone calls, $88(8.0 \%)$ by home visits and $63(5.7 \%)$ by hospital visits. In 58 (5.3\%) followups, the mode was not indicated. In relation to Day 14 follow-ups, $874(79.2 \%)$ were by telephone calls, with 88 $(8.0 \%)$ and $82(7.4 \%)$ being by way of home visits or in hospital, respectively. Day 21 follow-ups followed a similar pattern, with $932(84.5 \%)$ by telephone calls, $82(7.4 \%)$ by home visits and $38(3.4 \%)$ by hospital visits. In relation to $\mathrm{Hb}$ readings, there was marked differences between Ghana and Uganda. In the Ghana sites, baseline $\mathrm{Hb}$ was measured for 327 of the 360 patients, representing $90.8 \%$ of the patients. Seven patients in Ghana had $\mathrm{Hb}$ values recorded on both Day 0 and Day 14, and in all these cases the $\mathrm{Hb}$ values rose from baseline, indicating remission of anaemia. In Uganda, only 106 (14.3\%) of the 743 patients had Day $0 \mathrm{Hb}$ recorded and only one patient had Day 14 $\mathrm{Hb}$ recorded.

\subsection{Incidence of Any AEs}

The incidence of any AE by the end of follow-up among patients treated with Inj AS was estimated to be 17.9 (i.e. 197 of 1103) (95\% CI 15.8-20.3) (Table 1 and Fig. 2). The

Table 1 Incidence of any adverse events by baseline characteristics of patients, 2016

\begin{tabular}{|c|c|c|c|c|}
\hline Characteristics & Median (IQR) & Number of patients ( $\%$ of total) & $n(\%)$ who had any $\mathrm{AE}$ & $95 \% \mathrm{CI}$ \\
\hline \multicolumn{5}{|l|}{ Sex } \\
\hline Female & & $540(49.0)$ & $96(17.8)$ & $14.8-21.2$ \\
\hline Male & & $563(51.0)$ & $102(18.1)$ & $15.1-21.5$ \\
\hline Age (years) & $3.9(2,9)$ & & & \\
\hline$<5$ & & $654(59.3)$ & $115(17.6)$ & $14.8-20.7$ \\
\hline $5-9$ & & $186(16.9)$ & $24(12.9)$ & $8.8-18.5$ \\
\hline $10-19$ & & $61(5.5)$ & $10(16.4)$ & $9.0-27.9$ \\
\hline $15-19$ & & $40(3.6)$ & $10(25.0)$ & $14.0-40.6$ \\
\hline $20-24$ & & $46(4.2)$ & $10(21.7)$ & $12.1-35.9$ \\
\hline $25+$ & & $114(10.3)$ & $29(25.4)$ & $18.3-34.2$ \\
\hline Missing & & $2(0.2)$ & & \\
\hline Weight (kg) & $13(10,20)$ & & & \\
\hline$<10$ & & $255(23.1)$ & $49(19.2)$ & $14.8-24.5$ \\
\hline $10-19$ & & $470(42.6)$ & $72(15.3)$ & $12.3-18.9$ \\
\hline $20-29$ & & $105(9.5)$ & $14(13.3)$ & $8.1-21.3$ \\
\hline $30+$ & & $184(16.7)$ & $36(19.6)$ & $14.4-25.9$ \\
\hline Missing & & $89(8.1)$ & & \\
\hline Time-to-onset of AE (days) & $9(4,14)$ & & & \\
\hline \multicolumn{5}{|l|}{ Site } \\
\hline Ghana & & $360(32.6)$ & $125(16.8)$ & $14.3-19.7$ \\
\hline Uganda & & $743(67.4)$ & $73(20.3)$ & $16.4-24.8$ \\
\hline \multicolumn{5}{|l|}{ Pregnant } \\
\hline No & & $1067(96.7)$ & $193(18.1)$ & $15.9-20.5$ \\
\hline Yes & & $68(3.3)$ & $5(13.9)$ & $5.9-29.3$ \\
\hline Total & & $1103(100)$ & 197 (17.9) & $15.8-20.3$ \\
\hline
\end{tabular}

$A E$ adverse event, $C I$ confidence interval, $I Q R$ interquartile range 
Fig. 2 Proportion of patients with any adverse events by time, 2016: Kaplan-Meier failure estimate. $A E$ adverse event, $C I$ confidence interval

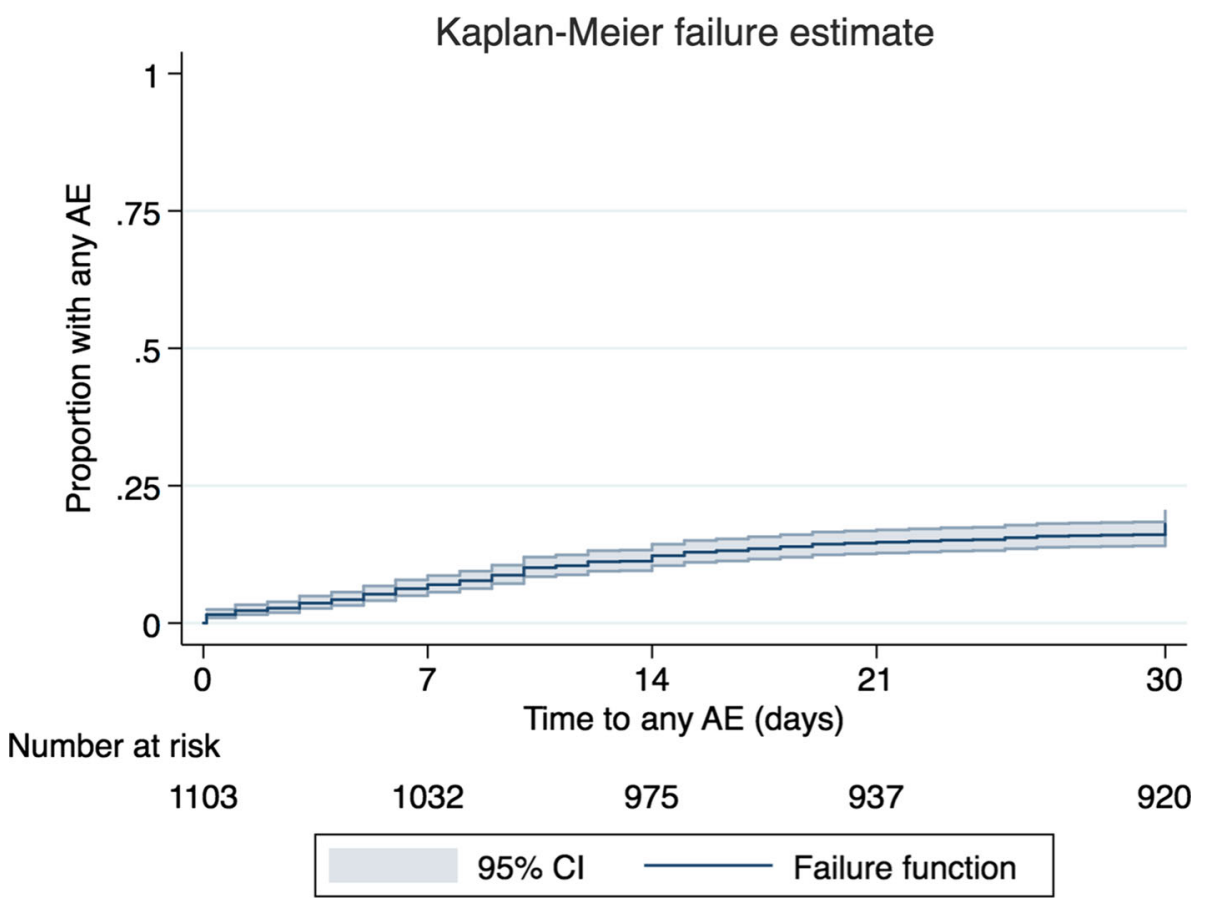

Table 2 Top five adverse events by sex and time-to-onset among patients treated with injectable artesunate at all sites, 2016

\begin{tabular}{|c|c|c|c|c|c|c|}
\hline & \multirow[t]{2}{*}{ Number of patients treated with Inj AS } & \multicolumn{5}{|c|}{$\mathrm{AE}[n(\%)]$} \\
\hline & & Pyrexia & Abdominal pain & Diarrhoea & Cough & Asthenia \\
\hline Sex & 1103 & & & & & \\
\hline Female & 540 & $16(3.0)$ & $13(2.4)$ & $6(1.1)$ & $9(1.7)$ & $8(1.5)$ \\
\hline Male & 563 & $22(3.9)$ & $14(2.5)$ & $13(2.3)$ & $7(1.2)$ & $8(1.4)$ \\
\hline Time-to-onset of AE (days) & 198 & & & & & \\
\hline $0-7$ & 77 & $11(14.3)$ & $12(15.6)$ & $6(7.8)$ & $5(6.5)$ & $9(11.7)$ \\
\hline $8-14$ & 58 & $12(20.7)$ & $4(6.9)$ & $8(13.8)$ & $5(8.6)$ & $6(10.3)$ \\
\hline $15-21$ & 27 & $7(25.9)$ & $2(7.4)$ & $3(11.1)$ & $4(14.8)$ & $1(3.7)$ \\
\hline $22-28$ & 36 & $8(22.2)$ & $9(25.0)$ & $2(5.6)$ & $2(5.6)$ & $0(0.0)$ \\
\hline Total & 1103 & $38(3.5)$ & $27(2.5)$ & $19(1.7)$ & $16(1.5)$ & $16(1.5)$ \\
\hline
\end{tabular}

$A E$ adverse event, Inj $A S$ injectable artesunate

median time-to-onset of any AEs was 9 days $(\mathrm{IQR}=4,14)$ (Table 1). The top five AEs recorded among patients treated with Inj AS were pyrexia (3.5\%), abdominal pain $(2.5 \%)$, diarrhoea $(1.7 \%)$, cough $(1.5 \%)$ and asthenia (1.5\%) (Table 2 and Fig. 3). Most of these top five AEs occurred in the first 14 days following treatment (Table 2). Regarding the relatedness of these AEs to Inj AS, 78.9\% of pyrexia (30/38), $63.0 \%$ of abdominal pain (17/27), 68.4\% of diarrhoea (13/19), $85.5 \%$ of cough (14/16) and $75.0 \%$ of asthenia (12/16) were assessed as 'possibly' related.

\subsection{SAEs Including Deaths}

During the study, 17 AEs were considered to be serious; 13 of these led to death. The deaths and the four other SAEs are described in Table 3. Four of the deaths occurred in seriously ill patients who were transferred from the hospital to their home with no further follow-up information due to the reluctance of carers/guardians and/or family members to provide any further information. Two others had no postmortem information even though follow-up information with family members confirmed death.

\section{Discussion}

This is the first large-scale post-approval safety study on Inj AS. It involved over 1100 patients who were exposed to at least one dose of Inj AS in the participating public health facilities in Ghana and Uganda. The majority of the 
Fig. 3 Adverse events among patients treated with injectable artesunate at all sites, 2016

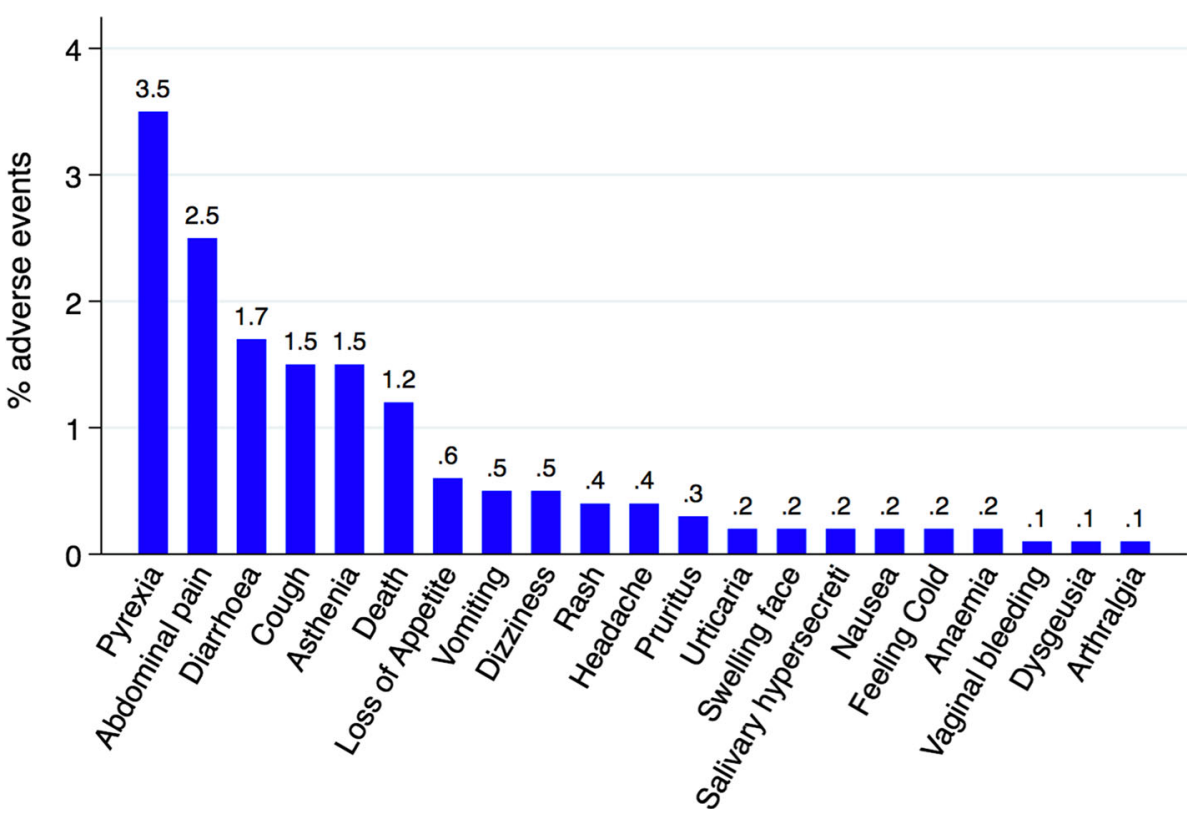

Table 3 Deaths and other serious adverse events reported in patients treated with injectable artesunate

\begin{tabular}{|c|c|c|}
\hline $\begin{array}{l}\text { Event } \\
\text { type }\end{array}$ & $n$ & Relationship to Inj AS intake \\
\hline Deaths & 13 & $\begin{array}{l}4 \text { of the } 13 \text { deaths did not have SAE specified and patients died outside the hospital with little information on follow-up. These } \\
\text { reports are classified as 'unassessable'. } 2 \text { of the remaining } 9 \text { fatal SAEs (severe anaemia in a } 22 \text {-month-old female and severe } \\
\text { anaemia in a } 20 \text {-month-old female) are causally assessed as 'possible' in relation to Inj AS intake. These SAEs are classified } \\
\text { as 'related' to Inj AS, though disease and other conditions could also explain these SAEs. The remaining } 7 \text { fatal SAEs (multi- } \\
\text { organ failure, severe respiratory distress, abdominal distension, asthenia, sickle cell disease, severe anaemia, pulmonary } \\
\text { tuberculosis) are unrelated to Inj AS intake }\end{array}$ \\
\hline $\begin{array}{l}\text { Other } \\
\text { SAEs }\end{array}$ & 4 & $\begin{array}{l}3 \text { of the } 4 \text { SAEs-severe abdominal pain in a 42-year-old female; failure of therapy and severe anaemia in a sickle cell disease } \\
\text { patient-are causally assessed as 'possible' in relation to Inj AS intake and thus related to Inj AS. } 1 \text { case-threatened } \\
\text { abortion-is considered to be causally assessed as 'unlikely' to be attributable Inj AS intake and is thus unrelated }\end{array}$ \\
\hline
\end{tabular}

Inj $A S$ injectable artesunate, $S A E$ serious adverse event

participants were children, with $59.3 \%$ being less than 5 years old. Inj AS was very well-tolerated among the study population even though nearly one-fifth of participants reported at least one mild to moderate AE. The most common AEs reported in both countries included pyrexia (3.5\%), abdominal pain (2.5\%), diarrhoea (1.7\%), cough $(1.5 \%)$ and asthenia $(1.5 \%)$. The relationship between Inj AS and most of these events were classified as 'possible' following case causality assessment. There were 13 allcause deaths reported in this study, giving an all-cause death rate of $1.2 \%$ (13/1103). Two of the deaths could be 'possibly' related to Inj AS. There were four other SAEs in addition to the deaths. Three of the non-fatal SAEs were 'possibly' related to Inj AS. Overall, the safety profile of Inj AS in the study population was favourable and comparable to that documented in the SEAQUAMAT and AQUAMAT studies.

The results obtained from this study are similar to the findings from clinical trials of Inj AS, including the
SEAQUAMAT and AQUAMAT studies. The overall incidence of $\mathrm{AE}$ is similar to that listed in the public assessment reports (PARs; Part 4: Summary of Product Characteristics) for Inj AS, as published by the WHO [27]. The PAR lists the following among the possible common (1-10 in 100 patients) AEs related to Inj AS: cough, diarrhoea, abdominal pain and 'flu-like' effects (including fever, tiredness, bone and muscle pain). These AEs are, however, also symptoms of malaria and severe malaria, making case causality assessment complex. Nonetheless, the study findings provide validation for the safety profile of Inj AS as recorded in the PAR. This study recorded a lower proportion of deaths than the AQUAMAT and SEAQUAMAT studies. In the AQUAMAT study, $8.5 \%$ of the 2712 patients in the artesunate arm died (230 African children), whilst $15 \%$ of the 730 patients in the artesunate arm of the SEAQUAMAT study died (107 Asian patients) $[4,5]$. It is important to state that, in contrast to our study, the SEAQUAMAT and AQUAMAT studies involved 
patients who had been clinically diagnosed with severe malaria. In our study we did not apply a strict definition of diagnosis of severe malaria as the aim was to follow patients who had been administered Inj AS in the 'normal course of clinical practice'. It is, therefore, possible that several of the cases in our study are not necessarily severe malaria, a serious disease with relatively high mortality. The lower mortality of $1.1 \%$ obtained in this study compares with a similar study [28] in Africa where an overall mortality of $1.03 \%(2 / 194)$ was recorded, though it must be stressed that reported mortality in severe malaria varies widely due to differences in practices, including not applying strict criteria for the definition of severe malaria [29]. Our study had two reports of severe anaemia which may be potential cases of PADH. PADH occurs 14 days after artesunate intake and has other features. However, the absence of pre-Day $14 \mathrm{Hb}$ readings in the two cases made a definite diagnosis of PADH fundamentally impossible.

This work has provided evidence indicating a favourable toxicity profile of Inj AS in real-world settings and one that is similar to that observed in earlier studies. However, it suffered from the limitations of most real-world studies. For instance, patients were enrolled if they had been administered at least one dose of Inj AS, presumably for the treatment of severe malaria. However, the majority of the patients were enrolled without any $\mathrm{Hb}$ readings and diagnosis of severe malaria was purely based on RDT and/ or microscopy. Even in cases where microscopy or RDT showed absence of malaria parasites, the patients were still administered Inj AS. The safety profile of Inj AS would not necessarily be expected to be different in patients with the potentially deadly severe malaria than in patients without severe malaria, though the outcomes of treatment may differ. Another limitation of the study was the absence of baseline and Day $14 \mathrm{Hb}$ values. Whilst there were 327 (91\%) Day $0 \mathrm{Hb}$ readings in Ghana, there were only seven (1.8\%) Day $14 \mathrm{Hb}$ readings in this same cohort. In Uganda, there were only 106 (14.3\%) Day $0 \mathrm{Hb}$ readings and one $(0.1 \%)$ Day $14 \mathrm{Hb}$ reading. Thus, in most of the participating facilities, most patients did not have more than one recorded $\mathrm{Hb}$ reading from the time of administration of Inj AS to the time the patient exited the study. This made it impossible to know whether there had been any drug-related changes in $\mathrm{Hb}$ values post-administration. Thus, even though very low levels of $\mathrm{Hb}$ were recorded in a few patients on Day 14, it was impossible to know whether this represented an existing severe anaemia or an actual fall due to Inj AS and which could thus have been a potential case of PADH. Another challenge in this real-world study was not being able to obtain follow-up information on four patients who died at home. The family/carers were not willing to provide any information, making it impossible to make any causal relationship. Finally, this study was not powered to detect rare AEs since the sample size of 1103 can only detect common AEs. It will be important to expand the study further in order to capture rare AEs in real-world settings. However, this follow-up study should, as a matter of ethics and for public health considerations, include a revision in the protocol for $\mathrm{Hb}$ readings to be made at baseline or soon thereafter and also at Day 14 to capture essential data to address the issue of PADH, which is a signal that has been raised in association with Inj AS use.

This study has provided additional information on the safety of Inj AS to that given in the pivotal studies that led to the WHO recommendation for its use as the medicine of choice in severe malaria. The incidence and types of AEs and SAEs observed in this study validates the WHO recommendation.

\section{Conclusion}

The incidence of common AEs among patients treated with Inj AS in real-world settings was relatively low. The overall safety profile of Inj AS among the treatment cohort was favourable. An interventional study to address PADH would be useful. Future studies should consider larger cohort to document rare AEs as well.

Acknowledgements The authors would like to thank the following for their support for the CEMISA research in the roles indicated:

African Collaborating Centre Accra, Ghana Team Members: Project Manager: Bernice Owusu-Boakye; IT Lead and Support: Danny Kofi-Armah and FelixJones Addo-Quaye; Site Coordinator: Alex Martey; Data Managers: Leticia Toffah and Prince N. Teye; Data Entry Operators: Samuel Larbi, Richard Narh Teye, Sandra Ampadu, Marilyn Amoama-Dapaah, Lawrencia Abrafi Osei and Richard Opata-Teye.

Sub Principal Investigators: Mame Yaa Nyarko (Princess Marie Louise Hospital, Ghana); Adriana Asante (Ridge Hospital, Ghana); Theresa Retigg (Agogo, Ghana); Madira Kefa (Kagadi Hospital, Uganda); Daniel Aguma (Lira Hospital, Uganda) Kizito John (Jinja Hospital, Uganda); and Komaketch Amal Denis (Mubende Regional Referral Hospital, Uganda)

Site Coordinators: Steven Tuure (Princess Marie Louise Hospital, Ghana); Mariam Mohammed (Ridge Hospital \& Princess Marie Louise Hospital, Ghana); Anthony Kwarteng (Kintampo Ghana); Martin Ssebidandi (Mubende Regional Referral Hospital, Uganda); Isaac Okorie (Lira Hospital, Uganda); Kabahuma Violet (Kagadi Hospital, Uganda); and Oboke Margaret (Jinja Hospital, Uganda).

Site Manager: Patrick Buabeng (Agogo, Ghana).

Advisor on Protocol and Study: Dr Stephan Duparc, Medicines for Malaria Venture.

\section{Compliance with Ethical Standards}

Conflicts of interest H. Hilda Ampadu, Alexander N.O. Dodoo, Samuel Bosomprah, Helga Gardarsdottir, H.G.M. Leufkens, Dan Kajungu and Kwaku Poku Asante have no conflicts of interest. Samantha Akakpo and Pierre Hugo are full-time employees of Medicines for Malaria Venture (MMV). 
Funding Medicines for Malaria Venture provided funding for this study.

Ethical approval The study received ethical approval from the Ghana Health Service Ethics Review Committee and the Uganda National Council for Science and Technology (UNCST). It was also registered on ClinicalTrials.gov with the ClinicalTrials.gov identifier NCT02817919. The study was conducted under Good Clinical Practice (GCP) guidelines taking into consideration the Declaration of Helsinki (as amended in October 2013) and local rules and regulations of participating countries and health facilities. All personnel involved in the study undertook and successfully passed an online GCP course prior to study initiation unless they already had a valid GCP certificate.

Patient consent Written informed consent was obtained from the patients for publication of this study. A copy of the written consent may be requested for review from the corresponding author.

Consent for publication Consent for publication was obtained as part of the informed consent process.

Open Access This article is distributed under the terms of the Creative Commons Attribution-NonCommercial 4.0 International License (http://creativecommons.org/licenses/by-nc/4.0/), which permits any noncommercial use, distribution, and reproduction in any medium, provided you give appropriate credit to the original author(s) and the source, provide a link to the Creative Commons license, and indicate if changes were made.

\section{References}

1. World Health Organization. World malaria report 2017. Geneva: World Health Organization; 2017. p. 41.

2. Olliaro P. Mortality associated with severe Plasmodium falciparum malaria increases with age. Clin Infect Dis. 2008;47(2):158-60.

3. World Health Organization. Guidelines for the treatment of malaria. 3rd ed. Geneva: World Health Organization; 2015.

4. Dondorp A, Nosten F, Stepniewska K, Day N, White N, South East Asian Quinine Artesunate Malaria Trial (SEAQUAMAT) Group. Artesunate versus quinine for treatment of severe falciparum malaria: a randomised trial. Lancet. 2005;366(9487):717-25.

5. Dondorp AM, Fanello CI, Hendriksen IC, Gomes E, Seni A, Chhaganlal KD, et al. Artesunate versus quinine in the treatment of severe falciparum malaria in African children (AQUAMAT): an open-label, randomised trial. Lancet. 2010;376(9753):1647-57.

6. Sinclair D, Donegan S, Isba R, Lalloo D. Artesunate versus quinine for treating severe malaria. Cochrane Database Syst Rev. 2012;6:CD005967.

7. Sinclair D, Donegan S, Lalloo D. Artesunate versus quinine for treating severe malaria. Cochrane Database Syst Rev. 2011;3:CD005967.

8. Li Q, Weina P. Artesunate: the best drug in the treatment of severe and complicated malaria. Pharmaceuticals. 2010;3(7):2322-32.

9. Taylor WRJ, White NJ. Antimalarial drug toxicity. Drug Saf. 2004;27(1):25-61.

10. Alkadi HO. Antimalarial drug toxicity: a review. Chemotherapy. 2007;53(6):385-91.

11. Rolling T, Wichmann D, Schmiedel S, Burchard GD, Kluge S, Cramer JP. Artesunate versus quinine in the treatment of severe imported malaria: comparative analysis of adverse events focussing on delayed haemolysis. Malar J. 2013;15(12):241. https://doi.org/10.1186/1475-2875-12-241.

12. Eder M, Farne H, Cargill T, Abbara A, Davidson RN. Intravenous artesunate versus intravenous quinine in the treatment of severe falciparum malaria: a retrospective evaluation from a UK centre. Pathog Glob Health. 2012;106(3):181-7. https://doi.org/10.1179/ 2047773212 Y.0000000032.

13. Krudsood S, Wilairatana $\mathrm{P}$, Vannaphan S, Treeprasertsuk S, Silachamroon U, Phomrattanaprapin W, et al. Clinical experience with intravenous quinine, intramuscular artemether and intravenous artesunate for the treatment of severe malaria in Thailand. Southeast Asian J Trop Med Public Health. 2003;34(1):54-61.

14. Ferrari G, Ntuku HM, Burri C, Tshefu AK, et al. An operational comparative study of quinine and artesunate for the treatment of severe malaria in hospitals and health centres in the Democratic Republic of Congo: the MATIAS study. Malar J. 2015;30(14):226. https://doi.org/10.1186/s12936-015-0732-1.

15. Ntuku HM, Ferrari G, Burri C, Tshefu AK, Kalemwa DM, Lengeler C. Feasibility and acceptability of injectable artesunate for the treatment of severe malaria in the Democratic Republic of Congo. Malar J. 2016;8(15):18. https://doi.org/10.1186/s12936015-1072-x.

16. Aldámiz-Echevarría Lois T, López-Polín A, Norman FF, MongeMaillo B, López-Vélez R, Perez-Molina JA. Delayed haemolysis secondary to treatment of severe malaria with intravenous artesunate: report on the experience of a referral centre for tropical infections in Spain. Travel Med Infect Dis. 2017;15:52-6.

17. Rolling T, Agbenyega T, Issifou S, Adegnika AA, Sylverken J, Spahlinger D, et al. Delayed hemolysis after treatment with parenteral artesunate in African children with severe malaria-a double-center prospective study. J Infect Dis. 2014;209(12):1921-8

18. Caramello P, Balbiano R, De blasi T, Chiriotto M, Deagostini M, Calleri G. Severe malaria, artesunate and haemolysis. J Antimicrob Chemother. 2012;67(8):2053-4.

19. Byakika-Kibwika P, Achan J, Lamorde M, Karera-Gonahasa C, et al. Intravenous artesunate plus Artemisnin based Combination Therapy (ACT) or intravenous quinine plus ACT for treatment of severe malaria in Ugandan children: a randomized controlled clinical trial. BMC Infect Dis. 2017 28;17(1):794. https://doi.org/ 10.1186/s12879-017-2924-5.

20. Roussel C, Caumes E, Thellier M, Ndour PA et al. Artesunate to treat severe malaria in travellers: review of efficacy and safety and practical implications. J Travel Med. 2017. https://doi.org/10. 1093/jtm/taw093.

21. Ampadu HH, Hoekman J, de Bruin ML, Pal SN, Olsson S, Sartori D, Leufkens HG, Dodoo AN. Adverse drug reaction reporting in Africa and a comparison of individual case safety report characteristics between Africa and the rest of the world: analyses of spontaneous reports in VigiBase $^{\circledR}$. Drug Saf. 2016;39(4):335-45. https://doi.org/10.1007/s40264-015-0387-4.

22. Kuemmerle A, Dodoo AN, Olsson S, Van Erps J, Burri C, Lalvani PS. Assessment of global reporting of adverse drug reactions for anti-malarials, including artemisinin-based combination therapy, to the WHO Programme for International Drug Monitoring. Malar J. 2011;9(10):57. https://doi.org/10.1186/14752875-10-57.

23. Layton D, Hazell L, Shakir SA. Modified prescription-event monitoring studies: a tool for pharmacovigilance and risk management. Drug Saf. 2011;34(12):e1-9. https://doi.org/10.2165/ 11593830-000000000-00000.

24. Layton D, Shakir SA. Specialist Cohort Event Monitoring studies: a new study method for risk management in pharmacovigilance. Drug Saf. 2015;38(2):153-63. 
25. Zwang J, D'Alessandro U, Ndiaye J-L, Djimdé AA, Dorsey G, Mårtensson AA, et al. Haemoglobin changes and risk of anaemia following treatment for uncomplicated falciparum malaria in subSaharan Africa. BMC Infect Dis. 2017;17(1):443.

26. Dodoo ANO, Fogg C, Nartey ET, Ferreira GLC, Adjei GO, Kudzi W, et al. Profile of adverse events in patients receiving treatment for malaria in urban Ghana: a cohort-event monitoring study. Drug Saf. 2014;37(6):433-48.

27. Summary of product characteristics: artesunate $60 \mathrm{mg}$ for injection. https://extranet.who.int/prequal/sites/default/files/ documents/MA051part4v2.pdf. Accessed 20 Jul 2017.
28. Kremsner PG, Taylor T, Issifou S, Kombila M, Chimalizeni Y, et al. A simplified intravenous artesunate regimen for severe malaria. J Infect Dis. 2012;205(2):312-9. https://doi.org/10.1093/ infdis/jir724.

29. Camponovo F, Bever CA, Galactionova K, Smith T, Penny MA. Incidence and admission rates for severe malaria and their impact on mortality in Africa. Malar J. 2017;16(1):1. https://doi.org/10. 1186/s12936-016-1650-6. 